\title{
Biologie et poétologie
}

José-Maria de Heredia, Leconte de Lisle et Ernst Haeckel

\section{Henning Hufnagel}

\section{CpenEdition}

\section{Journals}

Electronic version

URL: http://journals.openedition.org/aes/1621

DOI: 10.4000/aes.1621

ISSN: 2258-093X

\section{Publisher}

Laboratoire LISAA

Electronic reference

Henning Hufnagel, « Biologie et poétologie », Arts et Savoirs [Online], 9 | 2018, Online since 18 February 2019, connection on 01 May 2019. URL : http://journals.openedition.org/aes/1621 ; DOI : 10.4000/ aes. 1621

This text was automatically generated on 1 May 2019.

Centre de recherche LISAA (Littératures SAvoirs et Arts) 


\title{
Biologie et poétologie
}

José-Maria de Heredia, Leconte de Lisle et Ernst Haeckel

\author{
Henning Hufnagel
}

\section{AUTHOR'S NOTE}

Cet article a été conçu dans le cadre du programme ANR-DFG « Biolographes » (dir. Gisèle Séginger et Thomas Klinkert). Il s'agit d'une version développée de la troisième partie de mon article publié dans la revue en ligne Lendemains. Études comparées sur la France (n - 162/163, 2016, p. 64-82), sous le titre : „Zauberhafte Lichteffekte. Ästhetik und Wissenschaft bei Haeckel, Bölsche und Heredia“. http://periodicals.narr.de/index.php/ Lendemains/article/view/2937.

L'article paru dans Lendemains est une version abrégée du chapitre 7.4 de mon livre Wissen und Diskurshoheit. Zum Wissenschaftsbezug in Lyrik, Poetologie und Kritik des Parnasse 1840-1900 (Berlin/Boston, de Gruyter, 2017, p. 213-236). Je reprends de ce livre aussi l'analyse du poème Le Lac (p. 270-290). 
1 En histoire littéraire, la question des « influences » reste souvent - plus souvent que l'on ne voudrait - sans réponse définitive. On peut chercher à reconstruire la bibliothèque des écrivains, à tracer leurs lectures, à rétablir leurs réseaux sociaux et culturels, et dans de nombreux cas, ces recherches produisent des résultats impressionnants. Mais trop souvent, on

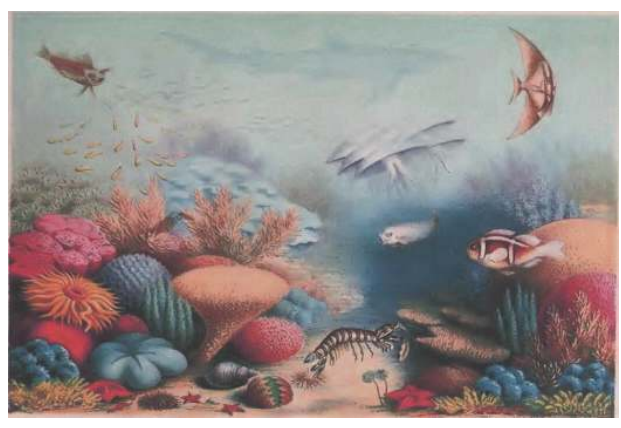
arrive au moment où force est de constater, en empruntant la formule célèbre d'Emil Heinrich Du Bois-Reymond: ignoramus, ignorabimus - nous ne savons pas et ne sauront probablement jamais si un tel a vraiment lu et connu tel ou tel texte.

2 Cela vaut pour tout matériau de "source ", et cela vaut particulièrement pour le savoir biologique au $\mathrm{XIX}^{\mathrm{e}}$ siècle, un savoir multiforme, rapidement croissant et souvent controversé, dont les éléments, «les termes et les représentations circulent abondamment entre les disciplines » et les domaines, des sciences aux « lettres » jusqu'à la politique ${ }^{1}$. Cette circulation est facilitée par le fait qu'en ces décennies-là, la biologie, en tant que discipline universitaire, est toujours en formation et en mouvement. Une telle situation épistémologique multiplie donc les canaux d'influence et d'intertextualité. En plus, ce savoir ne circule pas seulement d'un domaine à l'autre, mais une fois passé audelà du domaine scientifique, il circule aussi à l'intérieur du domaine littéraire. Les auteurs qui s'intéressent aux sciences de la nature empruntent des éléments non seulement aux ouvrages scientifiques, mais s'inspirent aussi des œuvres d'autres écrivains et de leurs représentations d'éléments biologiques. Entre eux, ils réfléchissent sur les nouveaux savoirs et discutent, de manière plus générale, les modalités d'une littérature qui opère en analogie avec la science - comme, par exemple, Flaubert et Leconte de Lisle ${ }^{2}$.

3 S'il faut donc constater la multiplicité des influences et se contenter de probabilités, il semble intéressant de modifier la question. Si on peut détecter un certain motif de savoir biologique dans un texte littéraire, il faut encore se demander de quelle manière et avec quel but l'auteur le (ré)utilise: quelle fonction revêt ce motif à l'intérieur de ce texte spécifique ? Et c'est exactement la question que je me suis proposé de poser en examinant deux poèmes de José-Maria de Heredia et de Leconte de Lisle. Je pose cette question à propos de deux motifs assez répandus dans la littérature de l'époque : le monde sousmarin des coraux ${ }^{3}$ et l'arbre comme symbole d'ordre et de développement ascendant. Or, ces motifs occupent aussi une place centrale dans l'œuvre d'Ernst Haeckel. Haeckel est une figure-clé du savoir biologique au XIX ${ }^{\mathrm{e}}$ siècle et de sa diffusion, et c'est pourquoi je confronte les deux poèmes à ses textes. Si je ne vise donc pas à "prouver » une relation de source et de cible (sans laisser complètement de côté cette question dans ma première partie), je montrerai que les deux poèmes témoignent néanmoins de l'impact, direct et indirect, du zoologue. 


\section{José-Maria de Heredia et les coraux arabes}

Dans les Trophées de José-Maria de Heredia, il y a un sonnet sur un récif de corail, qui, par sa thématique, sans fonds historique, fait plutôt exception dans le volume :

LE RÉCIF DE CORAIL

Le soleil sous la mer, mystérieuse aurore,

Éclaire la forêt des coraux abyssins

Qui mêle, aux profondeurs de ses tièdes bassins,

La bête épanouie et la vivante flore.

Et tout ce que le sel ou l'iode colore,

Mousse, algue chevelue, anémones, oursins,

Couvre de pourpre sombre, en somptueux dessins,

Le fond vermiculé du pâle madrépore.

De sa splendide écaille éteignant les émaux,

Un grand poisson navigue à travers les rameaux ;

Dans l'ombre transparente indolemment il rôde ;

Et, brusquement, d'un coup de sa nageoire en feu

Il fait, par le cristal morne, immobile et bleu,

Courir un frisson d'or, de nacre et d'émeraude. ${ }^{4}$

5 Les chercheurs ont longtemps estimé que Heredia s'est inspiré, pour ce poème publié pour la première fois en 1882, d'un ouvrage de Darwin, The Structure and Distribution of Coral Reefs (1842). Un exemplaire de la traduction française de 1878 se trouvait dans sa bibliothèque ${ }^{5}$. Darwin, pourtant, cherche à formuler une théorie sur la formation des récifs de corail, sujet qui ne joue aucun rôle dans le poème. Darwin ne s'intéresse guère à la classification zoologique et à l'apparence des coraux singuliers, qui sont les deux éléments qui forment les deux quatrains du sonnet de Heredia. Dans le second, Heredia thématise la coloration du récif, dans le premier, la position apparemment intermédiaire $\mathrm{du}$ corail entre faune et flore, par exemple quand il évoque, avec une construction syntactique raffinée, le corail comme de "La bête épanouie et la vivante flore ». Lorsque Darwin parle occasionnellement du statut de certains coraux, il les situe encore dans le règne végétal : «the Nulliporae undoubtedly belong to one of the lowest classes of the vegetable kingdom $"^{6}$. De surcroît, Heredia parle des coraux de la Mer Rouge - des coraux "abyssins ", avec une expression géographiquement un peu décalée, mais surtout plus précieuse $^{7}$. Darwin, en revanche, base son livre sur ses observations de récifs des Maldives et des îles Cocos ou Keeling et dans l'océan Indien ${ }^{8}$. Heredia a donc dû trouver son savoir biologique ailleurs. Mais où?

6 Or, si les Kunstformen der Natur de 1904 sont probablement le livre d'Ernst Haeckel le plus célèbre sur les animaux marins, c'est pourtant son dernier livre à ce sujet, après plusieurs autres, notamment sur les méduses et les radiolaires - et, à côté d'eux, un livre de vulgarisation sur les coraux de la Mer Rouge justement: Arabische Korallen. Ein Ausflug nach den Korallenbänken des Roten Meeres und ein Blick in das Leben der Korallentiere. Populäre Vorlesung mit wissenschaftlichen Erläuterungen ${ }^{9}$ de 1876.

7 Provoqué par l'affirmation peu pertinente qui désigne Darwin comme source de Heredia, j'aimerais confronter le texte de Heredia avec celui de Haeckel. On pourrait formuler l'hypothèse selon laquelle Heredia se serait inspiré de ce livre de Haeckel. Mais si, dans ce 
qui suit, j'explore les résonances possibles entre les deux textes, mon but n'est pas, comme j'ai déjà dit, de « prouver » une influence directe de Haeckel. Je confronte les deux textes l'un avec l'autre pour montrer comment Heredia reprend et re-fonctionnalise des motifs de savoir biologique et comment, de manière étonnante, un savant et un poète, un traité et un sonnet plus ou moins contemporains, se rejoignent ou se différencie. On verra que le poète n'opère pas simplement sous le signe d'une subjectivité esthétisante et que le livre du savant n'est pas une présentation objective de « faits » scientifiques.

Il n'est pas surprenant que les deux textes, le poème et le traité, parlent d'animaux marins, de coraux. Comme j'ai déjà laissé entendre, quand ils paraissent, le monde marin est un sujet à la mode dans la littérature, chez Michelet, Jules Verne et bien d'autres auteurs encore ${ }^{10}$. En effet, depuis les années 1860 , toute l'Europe subit la fascination du monde des océans, des profondeurs marines encore largement inexplorées. Cette fascination encourage l'établissement de larges aquariums publics dans toutes les grandes capitales du continent et résulte, peu après, dans l'introduction d'un nouveau « meuble » indispensable à chaque salon bourgeois : un petit « océan sous verre ». En l'Angleterre de l'époque, on parle d'un véritable "aquarium craze"11.

Ce qui est surprenant, toutefois, c'est le nombre et la précision apparente des résonances et des correspondances entre les deux textes. Pris isolément, chaque écho ne permet pas d'aboutir à des conclusions. Mais c'est leur ensemble qui suscite des interrogations.

Bien que je n'aie pas trouvé de traduction française, le livre allemand était présent à Paris; il y en a un exemplaire encore aujourd'hui à la Bibliothèque nationale de France ${ }^{12}$. Le manque d'une traduction n'empêche pas que les contemporains soient bien au courant des recherches de Haeckel sur les coraux, et non seulement les scientifiques, mais aussi les écrivains et les critiques comme Ferdinand Brunetière. En esquissant son propre programme d'une théorie évolutionniste de l'histoire littéraire, Brunetière déclare que, pour cette théorie, les objets de Haeckel en tant qu'objets biologiques n'ont pas d'importance: «Je ne m'intéresse guère aux récifs de corail, et peu de choses en soi me seraient plus indifférentes que les éponges calcaires $»^{13}$. Dans cette remarque, Brunetière fait allusion non seulement aux recherches de Haeckel sur les coraux, mais même à une deuxième œuvre de Haeckel non traduite en français. À côté de son livre sur les coraux de la Mer Rouge, en 1872, Haeckel avait publié une monographie en trois volumes sur les éponges calcaires ${ }^{14}$.

11 Vu que Heredia a acheté ou a reçu en cadeau le livre de Darwin sur les récifs de corail, quelqu'un dans son vaste réseau d'amis et de connaissances a pu lui signaler aussi le livre de Haeckel. Heredia était célèbre pour son hospitalité, et à la différence d'autres salons de poètes - comme ceux de Leconte de Lisle ou de Mallarmé, par exemple -, son salon n'était pas exclusivement centré sur les questions de littérature. Il accueillait aussi des historiens, des philologues, des hommes politiques et militaires, des scientifiques et des explorateurs ${ }^{15}$. Heredia semble avoir eu un penchant particulier pour ces derniers, ces " conquistadors" aux frontières du savoir. L'un d'entre eux était Maurice Maindron, zoologue, attaché au Muséum national d'histoire naturelle, qui dès les années 1870 entreprit de nombreux voyages d'exploration en Afrique et en Asie. Avec Heredia, il partageait aussi une fascination complètement différente : celle pour les armes antiques. Si Heredia écrivait des poèmes sur des épées somptueusement décorées, Maindron se plaisait à ciseler des gardes d'épées pendant son temps libre. ${ }^{16} \mathrm{En}$ effet, Maindron était très proche du poète et de sa famille : en 1899, il épousa la fille ainée de Heredia, Hélène. 
12 Maindron était spécialiste d'entomologie. Toutefois, ses connaissances et ses intérêts en zoologie dépassaient de loin cette discipline. Et tout au long de sa carrière, il visait particulièrement la vulgarisation des connaissances scientifiques. Il collabora à plusieurs encyclopédies et dictionnaires pour lesquels il écrivit de très nombreux articles, couvrant toute la gamme des sujets biologiques, par exemple dans le deuxième supplément du Grand dictionnaire universel $d u X_{X I X}{ }^{e}$ siècle ${ }^{17}$ ou le Nouveau Larousse illustré ${ }^{18}$. Si l'on voulait chercher un «relais » entre Heredia et le livre de Haeckel, Maurice Maindron serait le candidat le plus probable.

Or, quelles sont les résonances et correspondances que l'on peut relever entre les deux textes? D'abord, comme j'ai déjà indiqué, Heredia et Haeckel parlent tous les deux des coraux de la Mer Rouge, tandis que la «source» prétendue du sonnet, Darwin, observe des récifs d'une zone géographique complètement différente: l'océan Indien. Et si, contrairement à Heredia, Darwin situe certains coraux dans le règne végétal, comme on a vu plus haut, le livre de Haeckel s'ouvre justement sur une longue discussion à propos de la nature du corail entre plante et animal, pour enfin les situer fermement dans le règne animal, et des expressions comme „Pflanzenthiere“, «plantes-animaux » reviennent tout au long de l'ouvrage ${ }^{19}$.

14 Il y a encore d'autres mots-clés du sonnet qui sont aussi présents dans le texte de Haeckel. Par exemple, Heredia utilise « cristal » pour « eau »; Haeckel ne se lasse pas de décrire l'eau qui entoure le récif qu'il a exploré chaque fois qu'il en parle comme „krystallklar“ et „krystallhell ${ }^{“ 20}$. On trouve aussi des correspondances dans les éléments de la construction et dans la structuration du poème et du texte de Haeckel ; j'y reviendrai plus tard. Mais c'est surtout une illustration de la main de Haeckel lui-même, la vue d'ensemble d'un récif, et son commentaire qui invitent à être confrontés au sonnet de Heredia.

Ce commentaire se divise en trois parties de valeur épistémique différente, car tout le livre se présente comme un hybride : il a une dimension de vulgarisation qui vise le public général, mais aussi une dimension scientifique qui vise les collègues. Le texte principal se présente comme une vulgarisation didactique, puis l'on est renvoyé à des notes désignées explicitement comme scientifiques et enfin les deux côtés se mêlent dans un deuxième type de note, l'explication de l'illustration ${ }^{21}$. Dans les notes scientifiques, on lit sous le titre de „Farbenpracht der Korallenbänke“ :

Der wunderbare und unvergleichliche Farbenglanz der arabischen Korallen-Riffe, den kein Pinsel wiederzugeben vermag, erscheint zu verschiedenen Zeiten sehr verschieden. In seiner vollen Pracht erblickt man ihn um die Mittagszeit bei vollkommen stillem und klarem Wetter, wo die strahlende Mittagssonne ungebrochen durch das krystallhelle Wasser dringt und die stark mit Wasser geschwellten Korallenthiere ihren weichen Körper in voller Ruhe möglichst entfaltet haben. Wenn dagegen die Sonne unter spitzerem Winkel eintritt, wenn das Wasser getrübt oder etwas bewegt ist und wenn in Folge dessen die Korallenthiere sich zusammengezogen und Wasser abgegeben haben, so ist der zauberhafte Lichteffect nicht entfernt so schön. ${ }^{22}$

Observons d'abord que la note scientifique aussi est imprégnée de jugements esthétiques. Mais observons surtout les parallèles entre le passage de Haeckel et le sonnet de Heredia : tous les deux sont centrés sur les couleurs somptueuses du récif de corail; tous les deux thématisent plus spécifiquement un changement des couleurs, le changement de l'« effet de lumière enchanteur ». J'ai cherché à montrer dans une autre contribution comment Heredia recrée linguistiquement cet effet de lumière, particulièrement grâce au 
changement rapide des voyelles dans le dernier vers, où le «o" subit plusieurs transformations ${ }^{23}$.

Ce changement est lié, chez les deux auteurs, au contraste entre immobilité et mouvement. Haeckel ne parle pas de la source de ce mouvement ; chez Heredia, elle est l'élément central de la construction du sonnet, généralement caractérisée par une opposition dialectique entre les quatrains et les tercets. Tandis que les deux quatrains décrivent les coraux immobiles, les deux tercets fournissent l'élément de contraste : un grand poisson qui introduit le mouvement dans ce monde quasi-végétal. Par son mouvement, il produit des " effets de lumière enchanteurs ", changeants, qui lui sont tout propres.

Or, chez Haeckel, des poissons sont au premier plan dans le texte principal du livre auquel la note scientifique se réfère. En effet, ce passage réunit tous les éléments du sonnet et même leur distribution. Dans un premier paragraphe, le texte principal « populaire » décrit les coraux en réfléchissant à leur apparence ambiguë entre faune et flore $^{24}$; dans un deuxième paragraphe, Haeckel parle du „vielgestaltiges Thierleben“25, de la « vie animale multiforme » qui peuple le récif sous la forme de nombreuses espèces de poisson. Il met en évidence avant tout un poisson qui semble changer de couleur selon la manière dont la lumière le touche :

Unter ihnen [den Fischen] fällt uns vor Allen der sonderbare Halbmondfisch auf (Platax Ehrenbergii, Taf. III, rechts oben, XII.) Sein platt zusammengedrückter, sichelförmiger Körper, der je nach dem Lichtfalle bald in gelbgrünlichem Bronzeglanz, bald in prachtvollem Blau strahlt, ist oben in eine lange dreieckige gekrümmte Rückenflosse, unten in eine gleich Analflosse ausgezogen. ${ }^{26}$

Il change donc de couleur, tout comme le poisson du sonnet de Heredia, et il faut noter qu'il le fait de la même manière: en passant d'une couleur métallique à une couleur claire :

Et, brusquement, d'un coup de sa nageoire en feu

Il fait, par le cristal morne, immobile et bleu,

Courir un frisson d'or, de nacre et d'émeraude.

L'illustration à laquelle j'ai fait allusion ajoute encore d'autres correspondances ${ }^{27}$. Elle est d'autant plus importante pour le livre - je dirais même qu'elle est centrale -, parce qu'elle fournit la vue d'ensemble des éléments que Haeckel développe. 


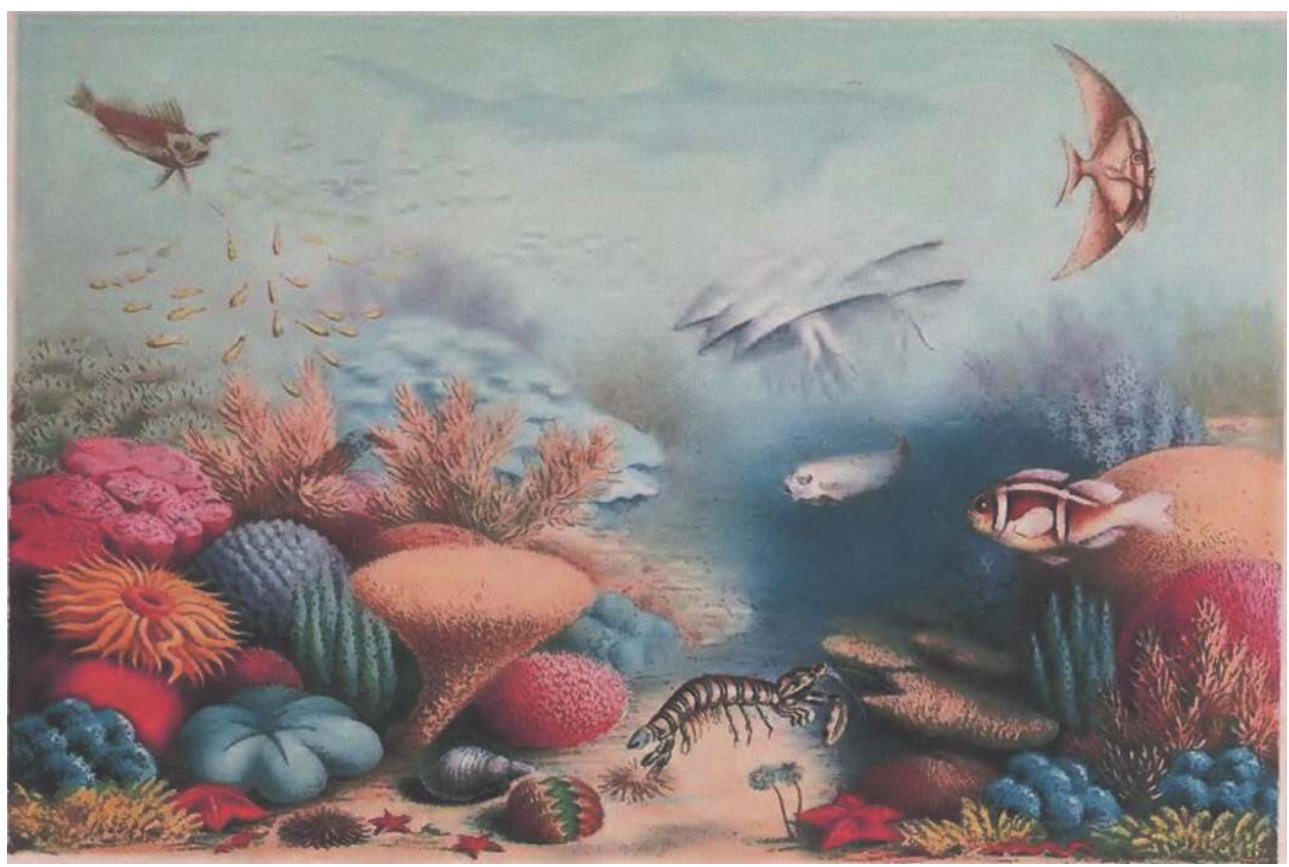

Berlin : Georg Reimer, 1876, Tafel III.

21 Au-dessus des coraux, Haeckel a peint plusieurs poissons de formes et de couleurs très différentes. Tout en haut, un poisson n'est qu'à peine visible; on dirait que, si le texte de Haeckel paraphrase quasiment le deuxième tercet du sonnet, l'image en illustre le premier :

De sa splendide écaille éteignant les émaux,

Un grand poisson navigue à travers les rameaux ;

Dans l'ombre transparente indolemment il rôde [...].

Si on compare l'image de Haeckel avec le sonnet de Heredia, le poème apparaît quasiment comme une transformation de l'illustration, traitée à la manière d'un tableau médiéval, représentant souvent - comme on sait - l'une à côté de l'autre, des actions qui se succèdent chronologiquement. Les poissons de Haeckel qui, l'un à côté de l'autre, présentent des couleurs différentes allant de l'ombre presque invisible jusqu'à un éclat aveuglant, sont transformés en un seul poisson chez Heredia, qui présente les mêmes changements de manière chronologique.

Si l'on a pu constater les correspondances détaillées entre les deux textes, leur lecture parallèle met aussi clairement en lumière les différences des procédés des deux auteurs, et cela de manière très parlante. Chez Haeckel, la présentation des matériaux scientifiques est soumise à une perspective esthétique, individuelle et personnelle. Elle est liée à l'expérience vécue par un individu, Haeckel qui plonge lui-même dans la mer et témoigne directement de la beauté des coraux : c'est la perspective d'un témoin qui sert à confirmer la vérité du discours ${ }^{28}$. Heredia, d'une part, vise à des effets de virtuosité artistique ; c'est pourquoi il sélectionne rigoureusement les données possibles. De toutes les couleurs du récif que Haeckel énumère, dans le sonnet, il ne reste que le pourpre pour mieux contraster avec les couleurs du poisson qui culminent dans un vert émeraude brillant. D'autre part, Heredia imite les procédés scientifiques. Il prend grand soin d'éliminer les traces d'une observation subjective, surtout en refusant tout indice qui 
pourrait servir à une interprétation allégorique de sa description. Grâce à cette écriture impersonnelle aux accents scientifiques, Heredia cherche à recréer les « effets de lumière enchanteurs " qui ont une valeur intrinsèque, qui peuvent donc rester des effets de lumière, sans avoir besoin d'une interprétation ${ }^{29}$. Et tandis que Haeckel souligne plusieurs fois que la réalité dépasse tous les essais de description, Heredia fait rivaliser les sonorités de la langue avec les impressions visuelles. Si Haeckel présente les merveilles de la nature, déployant des savoirs de manière didactique, Heredia met en place une poétologie de la virtuosité qui vise à émerveiller les lecteurs avec ses artifices : il y utilise des savoirs, mais, en n'offrant nulle piste d'interprétation allégorique, leur ôte toute dimension didactique.

\section{Leconte de Lisle, le lac et l'arbre} volumes, et c'est la raison pour laquelle il n'a pas retenu l'attention des interprètes. Dans sa nouvelle édition des poèmes tardifs de Leconte de Lisle, Edgar Pich écrit même : «Cette pièce n'a encore fait l'objet, à notre connaissance, d'aucun commentaire $»^{30}$. Le poème a été publié pour la première fois dans la Revue des Deux Mondes, c'est-à-dire dans une revue à grand public, en 1888, au moment où Leconte de Lisle venait de prendre la place de Victor Hugo à l'Académie. ${ }^{31}$ Ces deux aspects - la publication devant le grand public et consécration récente du poète - donnent donc au poème une importance majeure comme prise de position poétologique.

LE LAC

C'est une mer, un Lac blême, maculé d'îles Sombres, et pullulant de vastes crocodiles Qui troublent l'eau sinistre et qui claquent des dents. Quand la nuit morne exhale et déroule sa brume, Un brusque tourbillon de moustiques stridents Sort de la fange chaude et de l'herbe qui fume, Et dans l'air alourdi vibre par millions ; Tandis que, çà et là, panthères et lions, À travers l'épaisseur de la broussaille noire, Gorgés de chair vivante et le mufle sanglant, À l'heure où le désert sommeille, viennent boire ; Les unes en rasant la terre, et miaulant De soif et de plaisir, et ceux-ci d'un pas lent, Dédaigneux d'éveiller les reptiles voraces, Ou d'entendre, parmi le fouillis des roseaux, L'hippopotame obèse aux palpitants naseaux, Qui se vautre et qui ronfle, et de ses pattes grasses Mêle la vase infecte à l'écume des eaux.

Loin du bord, du milieu des roches erratiques, Solitaire, dressant au ciel son large front, Quelque vieux baobab, témoin des temps antiques, Tord les muscles noueux de l'immuable tronc Et prolonge l'informe ampleur de sa ramure 
Qu'aucun vent furieux ne courbe ni ne rompt,

Mais qu'il emplit parfois d'un vague et long murmure.

Et sur le sol visqueux, hérissé de blocs lourds,

Saturé d'âcre arome et d'odeurs insalubres,

Sur cette mer livide et ces îles lugubres,

Sans relâche et sans fin, semble planer toujours

Un silence de mort fait de mille bruits sourds. ${ }^{32}$

Quel tableau charmant de la nature! En trente alexandrins, Leconte de Lisle développe la description d'un lac et de ses alentours. On pourrait dire qu'il s'agit là d'un sujet poétique par excellence, voire topique. C'est déjà un premier indice montrant que nous avons à faire à un poème poétologique, d'autant plus que cette description semble réunir tous les éléments du lieu poétique par excellence, du locus amoenus ${ }^{33}$, pour en donner une version complètement renversée. Il semble que Leconte de Lisle veut raturer tous les attributs de la «belle nature». Au lieu d'une source ou d'un ruisseau - c'est-à-dire de l'eau en mouvement, qui coule, fraîche et peu abondante, Leconte de Lisle nous donne l'eau stagnante d'un lac aux dimensions gigantesques - «C'est une mer »-, rempli d'« eau sinistre ", "blême", écumeux, bordé de "vase infecte" qui exhale des "odeurs insalubres ». Au lieu de rives en fleurs et de prés, nous trouvons un « sol visqueux », «la fange chaude ", «l'herbe qui fume » et "la broussaille». La petite forêt qui invite à se reposer à l'ombre est remplacée par un arbre " solitaire », « informe », au nom barbare et qui par sa grandeur dépasse toutes les mesures, comme le fait également le lac dont il occupe le centre inaccessible. Enfin, le locus amoenus classique est habité par un monde animal paisible, d'une paix édénique, gardé très souvent par des bergers bucoliques. Chez Leconte de Lisle, en revanche, les animaux sont pour la plupart des prédateurs et tous se surpassent en gestes agressifs, des «moustiques stridents " aux "reptiles voraces » en passant par les panthères et lions au «mufle sanglant ». L'homme est complètement absent du poème, l'instance d'énonciation reste impersonnelle.

Le poème de Leconte de Lisle ne s'en prend pourtant pas à des traditions poétiques en général. Il mène son attaque très spécifiquement contre le romantisme, et ceci dès le titre : «Le Lac ». En lisant un tel titre, le lecteur peut penser à un poème beaucoup plus célèbre que celui de Leconte de Lisle : «Le Lac » (1817) d'Alphonse de Lamartine. On sait bien que Lamartine était une des "bêtes noires" de Leconte de Lisle. Mais son attaque poétologique n'est pas (ou pas seulement) personnelle. Bien qu'écrit soixante-dix ans plus tôt que le poème parnassien, «Le Lac » de Lamartine continue d'être perçu à la fin du siècle comme un des poèmes paradigmatiques du romantisme. En est la preuve le commentaire que Rémy de Gourmont fait à propos des «mérites » des Parnassiens en général, c'est-à-dire à propos de leur poétique antiromantique :

Ce mouvement nous délivra très heureusement des lacs poétiques aux flots harmonieux, des jeunes filles pâles au regard fatal, des jeunes hommes larmoyants, des faux désespérés dans le genre de celui qui exalte, dans la Nuit d'octobre, des plaintes si naïves. ${ }^{34}$

Si dans la deuxième partie de la phrase, la référence à Alfred de Musset est explicitée par le titre de la Nuit d'octobre, la référence à Lamartine dans la première n'est pas moins claire: les "flots harmonieux " sont une citation directe de la quatrième strophe du « Lac ». Là, le poète s'adresse au lac lui-même :

On n'entendait au loin, sur l'onde et sous les cieux,

Que le bruit des rameurs qui frappaient en cadence Tes flots harmonieux. ${ }^{35}$ 

poétique romantique qui en est le fondement, grâce principalement aux éléments biologiques que Leconte de Lisle insère dans son texte.

«Le Lac » de Lamartine est notamment une longue apostrophe élégiaque du poète au lac où il a passé une soirée avec sa bien-aimée. Il déplore la fuite du temps et la caducité de tout ce qui est humain : « Ne pourrons-nous jamais sur l'océan des âges / Jeter l'ancre un seul jour?» Il se rappelle que la bien-aimée l'avait justement exhorté à vivre dans le moment : «Aimons donc, aimons donc! de l'heure fugitive, / Hâtons nous, jouissons! » Un an après cette soirée, il est revenu seul au même endroit et évoque son souvenir d'elle en tant qu'inscrit dans la nature. Le poème finit sur cette strophe :

Que le vent qui gémit, le roseau qui soupire,

Que les parfums légers de ton air embaumé,

Que tout ce qu'on entend, l'on voit ou l'on respire, Tout dise : Ils ont aimé ! ${ }^{36}$

Le « Lac » de Lamartine présente donc des principes centraux de la poétique romantique : premièrement, au centre du poème il se passe un événement qui est présenté comme l'expérience personnelle du poète qui parle en son nom propre; deuxièmement, l'homme communique avec la nature, car la nature est centrée sur l'homme ${ }^{37}$ : elle existe pour que l'homme puisse y lire sa propre histoire.

Déjà sur le plan formel, les deux « Lacs » s'opposent. Le poème de Lamartine est composé de seize strophes à quatre vers, trois alexandrins suivis d'un vers de six syllabes, un sixain. Dans les strophes 6 à 9, le poète donne la parole à la bien-aimée elle-même; ces strophes combinent deux alexandrins avec deux sixains. Cette architecture des strophes est assez raffinée:le sixain, faisant quasiment figure d'un hémistiche d'alexandrin, évoque en même temps le vide causé par la personne absente et l'écho que la nature peut donner aux paroles du poète. Le poème de Leconte de Lisle, en revanche, consiste en une suite de trente alexandrins, divisés en deux groupes inégaux; les rimes faisant alterner rimes plates et rimes croisées ne suivent pas de schéma constant : après une alternance de deux rimes plates et de quatre rimes croisées, ce schéma est abandonné à partir du treizième vers. C'est donc une forme aussi «barbare» que la scène décrite. Chez Lamartine, la nature est dénommée "belle nature » (v. 51), tandis que nous avons vu comment Leconte de Lisle met en scène un renversement de ce topos.

Même si les deux poèmes parlent d'un soir et d'une nuit, les deux scènes ne pourraient être plus différentes. Leconte de Lisle semble avoir pris méticuleusement soin de mettre les détails en opposition : si le lac de Lamartine est entouré de "rocs sauvages » (v. 55), dans son poème, les « roches erratiques » se trouvent sur une île au milieu du lac. Et si le poème de Lamartine présente une forêt de «noirs sapins » européens, chez Leconte de Lisle, comme nous l'avons déjà vu, il n'y a qu'un seul arbre, le gigantesque baobab exotique.

Mais la différence la plus importante concerne les voix : chez Lamartine, c'est la voix humaine qui s'élève, d'abord celle du poète qui communique avec la nature directement, et puis celle de la bien-aimée qui parle pendant la nuit rappelée. Comme nous l'avons vu, chez Leconte de Lisle, il n'y pas de "je»; l'énonciateur reste, pour ainsi dire, extradiégétique à la scène décrite. Et s'il n'y a pas d'hommes dans ce poème, dans le poème de Lamartine, il n'y a pas d'animaux. Dans le «Lac » de Leconte de Lisle, toute une gamme de bruits animaux se fait entendre: les crocodiles "claquent des dents", les moustiques font un bruit strident, les panthères miaulent, l'hippopotame ronfle. Leconte 
de Lisle leur donne donc la place que l'homme avait dans le poème de Lamartine ; il nie la position centrale que celui-là y avait occupée en lui substituant des éléments de savoir biologique suggérés par l'histoire naturelle: des noms précis, des comportements et des habitats encore peu évoqués dans les bestiaires poétiques.

Or, ces animaux ne sont pas choisis arbitrairement. Pour une grande partie, ce sont des animaux poétologiquement importants pour Leconte de Lisle: ils font référence à quelques-uns de ses poèmes les plus célèbres des Poèmes barbares, notamment «Le Rêve du jaguar » où l'on rencontre le « mufle béant par la soif alourdi " ${ }^{38}$, « Les jungles » où une panthère «miaule tristement » en se dressant vers des " cours d'eau " ${ }^{39}$ et « L'Oasis » où l'on rencontre des lions et des hippopotames, eux aussi près de l'eau ${ }^{40}$. Ces trois poèmes partagent, par ailleurs, le même schéma formel avec « Le Lac » : ils consistent en une série d'alexandrins sans strophes régulières. Leconte de Lisle ne substitue donc pas seulement les animaux à l'homme; il substitue à cet élément de la poétique romantique des références à ses propres œuvres, en affirmant sa propre poétique.

L'élimination de l'homme implique un changement de perspective. Si «Le Lac» de Lamartine est centré sur un événement particulièrement présent encore dans l'exhortation au carpe diem de la bien-aimée, «Le Lac» de Leconte de Lisle souligne la continuité, la répétition, la récurrence éternelles des mêmes actions. Le poème utilise un présent intemporel. À cette focalisation sur la répétition correspond aussi la structure circulaire du poème: le premier vers - «C'est une mer, un Lac blême, maculé d'îles / sombres" - revient, à travers un lexique varié, mais sémantiquement identique, en ouverture de la dernière phrase du poème, au vers $27:$ «Sur cette mer livide et ces îles lugubres ».

L'élément poétologiquement le plus significatif de cette élimination de l'homme se trouve à la fin du poème, de nouveau en opposition directe à Lamartine. Son poème culmine dans les quatre dernières strophes : elles mettent en scène l'attribution à la nature d'un sens humain, dont les éléments sont interpelés un à un, avec un grand effet rhétorique, et chargé du souvenir du poète. Ironiquement, on pourrait dire que, tel un randonneur insouciant, il laisse l'image d'un cœur dans l'écorce de tous les arbres et sur tous les rochers devant lesquels il passe :

ô lac! rochers muets ! grottes ! forêt obscure !

Vous, que le temps épargne ou qu'il peut rajeunir,

Gardez de cette nuit, gardez, belle nature,

Au moins le souvenir!

Qu'il soit dans ton repos, qu'il soit dans tes orages, Beau lac, et dans l'aspect de tes riants coteaux, Et dans ces noirs sapins, et dans ces rocs sauvages Qui pendent sur tes eaux.

Qu'il soit dans le zéphyr qui frémit et qui passe, Dans les bruits de tes bords par tes bords répétés, Dans l'astre au front d'argent qui blanchit ta surface De ses molles clartés.

Que le vent qui gémit, le roseau qui soupire, Que les parfums légers de ton air embaumé, Que tout ce qu'on entend, l'on voit ou l'on respire, Tout dise : Ils ont aimé ! ${ }^{41}$ 
Notons que Lamartine fait appel à plusieurs sens : auditif, visuel et olfactif. Ce sont les mêmes avec lesquels Leconte de Lisle construit la fin de son poème, en renversant le poème romantique. $\mathrm{Si}$, chez Lamartine, un astre illumine le lac, chez Leconte, la scène reste dans une demi-obscurité menaçante, « livide » et « lugubre »; si chez Lamartine, le vent gémit, chez Leconte de Lisle, le « vent furieux » ne laisse naître qu'un « vague et long murmure »; si, chez Lamartine, l'air est « embaumé » de " parfums légers », chez Leconte de Lisle, on sent un « âcre arome » et des » odeurs insalubres ». Si enfin, chez Lamartine, la nature est le témoin de l'amour de deux êtres humains - et garantit la survie de quelques aspects de leur vie individuelle -, chez Leconte de Lisle, la nature, sous la forme du baobab, est certainement "témoin des temps antiques ", mais elle n'est que le témoin d'elle-même, de la répétition de ses processus, du retour éternel du même, n'incluant rien d'humain. Chez Lamartine, la nature est remplie de sens, de sens humain; chez Leconte, elle en est complètement vide : c'est pourquoi le poème finit sur un silence paradoxal, sur «Un silence de mort fait de mille bruits sourds »: la nature est incompréhensible, n'a rien à dire à l'homme, à l'homme en tant qu'individu, rien que sa mort.

On peut donc conclure que Leconte de Lisle - qui d'ailleurs se piquait d'érudition scientifique $^{42}$ - a appris la première leçon de l'évolutionnisme : l'homme n'est pas au centre de la nature ; il n'est qu'un accident de l'évolution. Vu la manière dont Leconte de Lisle présente l'arbre - le baobab impénétrable -, on peut conclure qu'il en a appris aussi la seconde leçon, encore plus importante, notamment qu'il n'y a pas de sens, pas de téléologie dans la nature. Tout comme Leconte de Lisle renverse les éléments centraux de la poétique romantique, il renverse aussi le symbole de l'arbre, symbole puissant d'ordre et de développement ascendant dont Jean-Louis Cabanès a souligné l'importance dans la littérature à la fin du $\mathrm{XIX}^{\mathrm{e}}$ siècle ${ }^{43}$. Il y est largement répandu comme image de la continuité de la vie et de l'épanouissement de l'énergie vitale. Même l'arbre généalogique des Rougon-Macquart qui est censé montrer l'épuisement et la dégénérescence de la famille, sous les yeux du Docteur Pascal, revêt soudainement un caractère optimiste :

[...] ce sont là les rameaux derniers de l'arbre, les dernières tiges pâles où la sève puissante des grosses branches ne semble pas pouvoir monter. Le ver était dans le tronc, il est à présent dans le fruit et le dévore... Mail il ne faut jamais désespérer, les familles sont l'éternel devenir. Elles plongent, au-delà de l'ancêtre commun, à travers les couches insondables des races qui ont vécu, jusqu'au premier être; et elles pousseront sans fin, elles s'étaleront, se ramifieront à l'infini, au fond des âges futurs... Regarde notre arbre : il ne compte que cinq générations, il n'a pas même l'importance d'un brin d'herbe, au milieu de la forêt humaine, colossale et noire, dont les peuples sont les grands chênes séculaires. ${ }^{44}$

40 La forme emblématique de ces arbres optimistes de « fin de siècle " se trouve dans les livres de Haeckel qui, dès la Generelle Morphologie der Organismen (1866), parsème ses œuvres en grand nombre d'arbres généalogiques : ils montrent comment, par le progrès de l'évolution, les êtres deviennent de plus en plus complexes, et, en même temps, de plus en plus beaux ${ }^{45}$. Dans son essai poétologique Die naturwissenschaftlichen Grundlagen der Poesie (1887), Wilhelm Bölsche emploie la métaphore de l'arbre dans le même sens pour montrer le progrès de l'évolution du protozoaire, en passant par les animaux pluricellulaires - Bölsche parle de „Zellenstaat“, «états cellulaires » - jusqu'à l'homme comme le „höchste und vollendetste Zellenstaat", « le plus haut et plus parfait des états cellulaires. $»^{46}$

41 Le baobab de Leconte de Lisle est la négation de tels arbres généalogiques à sens ascendant. Il n'y pas de développement, de progrès dans cet Éden à l'envers que Leconte 
de Lisle peint avec son lac primordial africain. Les animaux sont présentés sans ordre ; les représentants des classes différentes, des reptiles, insectes et mammifères, alternent de manière chaotique. En fait, si cette nature suivait un ordre ascendant, on aurait attendu de lire d'abord des vers sur la flore, puis sur la faune. Mais c'est seulement à la deuxième strophe qu'apparait le baobab, tandis que les animaux en sont complètement absents. Ces derniers paraissent eux-mêmes sans but; leurs actions restent pour la plupart sans objectif clair ${ }^{47}$. C'est un monde qui correspond bien au "message» de son arbre généalogique, du baobab en tant que "témoin des temps antiques»: un message indéchiffrable et chaotique.

\section{Conclusion}

Pour mettre encore plus en évidence la manière dont Heredia et Leconte de Lisle refonctionnalisent les savoirs biologiques, j'aimerais les confronter brièvement avec l'esthétique littéraire haeckelienne de Wilhelm Bölsche que j'ai analysée dans ma première contribution à ce dossier. Comparés à Haeckel et Bölsche, Heredia et Leconte de Lisle procèdent de manière très différente.

Heredia s'informe, il prend attentivement connaissance des nouveaux savoirs, tout comme Bölsche l'avait revendiqué. D'ailleurs, au même moment, Ferdinand Brunetière exige des poètes une attitude tout à fait comparable ${ }^{48}$. Mais, pour Heredia, la fidélité aux faits établis par la science n'est importante que jusqu'à un certain degré. La stylisation artistique est encore plus importante. Heredia laisse de côté des faits pour obtenir des effets esthétiques plus forts - par exemple, comme nous l'avons vu plus haut, dans son sonnet, il réduit les couleurs à une sorte de dichotomie, pour accentuer une structuration esthétique. Il revendique donc une marge de manœuvre fictionnelle et « rivalise » avec la science sur le plan esthétique plutôt qu'en cherchant à la corroborer esthétiquement, ce qui était le rôle que Haeckel avait prévu pour l'art. De plus, chez Heredia, tout énoncé sur la signification des situations et des actions relatées reste, tout au plus, implicite, sinon complètement absent.

Chez Leconte de Lisle aussi, on cherche en vain toute référence à une «bonne évolution » à la manière de Bölsche. Mais Leconte de Lisle est beaucoup plus explicite que Heredia, chose qui est due au fait que son but principal est le démontage de la poétique romantique. Leconte de Lisle entraîne aussi l'évolutionnisme ascendant à la manière de Haeckel dans ce démontage du romantisme: cet évolutionnisme en est une victime collatérale, pour ainsi dire ${ }^{49}$. Par son renversement systématique de certains topoï littéraires de couleur romantique, Leconte de Lisle nous présente, au lieu d'un développement vers la beauté, un Éden catastrophique. 


\section{NOTES}

1. Nadège Lechevrel, Bénédicte Percheron, Gisèle Séginger, «Billet 1. Le champ sémantique du Polype : expérimentations numériques », https://biolog.hypotheses.org/category/documents-etoeuvres/le-polype-formes-et-savoirs.

2. Leurs échanges et influences mutuelles apparaissent dans la correspondance de Flaubert, non pas dans des lettres à Leconte de Lisle lui-même (il n'y a que quelques billets adressés au poète qui nous sont parvenus, datant de la dernière décennie de la vie de Flaubert), mais surtout par le biais des lettres à Louise Colet dans les années 1853-1854. Voir Henning Hufnagel, «Flaubert parnassien, Parnasse flaubertien. Correspondances poétologiques ", in Julie Anselmini/Brigitte Diaz/Franziska Meier (dir.), Correspondances et critique littéraire, Paris, Garnier (à paraître).

3. Voir à propos du «corail» les analyses numériques de la récurrence du champ lexical «polype» qui inclut aussi «corail» Lechevrel, Percheron, Séginger, «Billet 1. Le champ sémantique du Polype », op. cit.

4. José-Maria de Heredia, Les Trophées, éd. Anny Detalle, Paris, Gallimard, 1981, p. 154.

5. Voir ibid., p. 328-329.

6. Charles Darwin, The Works of Charles Darwin, vol. 7, The Geology of the Voyage of H.M.S. Beagle. Part 1, Structure and Distribution of Coral Reefs, éd. Paul H. Barrett, New York, New York University Press, 1987, p. 11.

7. Quelqu'un pourrait être tenté de voir, dans ce mot d' "abyssin », non seulement une référence au pays africain, mais aussi une allusion aux abysses. Mais c'est une fausse piste : le Petit Robert fait une nette différence entre « abyssal » qui vient « des abysses » et « abyssin » qui désigne la provenance d'Abyssinie. En outre, cette différence confirme le fait que José-Maria de Heredia est informé des savoirs biologiques: les coraux se trouvent généralement dans les eaux peu profondes.

8. Voir Darwin, Structure and Distribution of coral reefs, op. cit. Son livre s'ouvre sur une longue description de la forme et de la structure des récifs de ces deux archipels classés comme " atolls » pour ensuite décrire d'autres types de récifs, surtout dans les océans Pacifique et Indien, suivant l'itinéraire du Beagle. Le seul passage où Darwin traite de la Mer Rouge de manière plus approfondie se trouve dans l'annexe où il synthétise des informations réunies par d'autres chercheurs et où il se focalise exclusivement sur la structure des récifs (ibid., p. 173-177).

9. Berlin, Georg Reimer, 1876. En français : Les Coraux arabes. Une excursion aux récifs de corail de la Mer Rouge et un regard sur la vie des animaux-coraux. Leçon populaire avec des explications scientifiques.

10. Voir à propos du motif du "polype " dans la littérature du XIX siècle particulièrement Nadège Lechevrel, Bénédicte Percheron, Gisèle Séginger, « Billet 5. Le Polype littéraire », https:// biolog.hypotheses.org/2164.

11. Voir Thomas Brandstetter

Brandstetter, Thomas

/Christina Wessely

Wessely, Christina

, « Einleitung : Mobilis in mobili », in Berichte zur Wissenschaftsgeschichte, 36, 2013,

p. 119-127, ici p. 119-120. Pour l'histoire culturelle de l'aquarium voir aussi Bernd Brunner

Brunner, Bernd 
, Wie das Meer nach Hause kam. Die Erfindung des Aquariums, Berlin, Transit, 2003).Voir Hufnagel, Wissen und Diskurshoheit, op. cit., p. 208.

12. Cote : FOL-S-12.

13. Ferdinand Brunetière, Évolution des genres dans l'histoire de la littérature [1890], Paris, Hachette, 1914, p. 200.

14. Voir Ernst Haeckel

Haeckel, Ernst

, Die Kalkschwämme - Calcispongae, 3 vols., Berlin, Reimer, 1872.

15. Voir Dominque Bona, Les yeux noirs. Les vies extraordinaires des sœurs Heredia, Paris, Clattès, 1989, p. 54-55. Elle nomme, entre autres, Gabriel Hanoteaux, Jean Psichari, Auguste Angellier, Alfred Dodds et Edouard Foa.

16. Voir Heredia, Les Trophées, op. cit., p. 128 ; voir la nécrologie de Maurice Maindron dans le Larousse mensuel illustré. Revue encyclopédique universelle, publiée sous la direction de Claude Augé, vol. 2, Paris, Larousse, 1911-1913, p. 212.

17. Dans la note sur les collaborateurs à la fin du deuxième supplément, on lit: « M. Maurice Maindron, qui a exploré en naturaliste l'archipel Malais et la Nouvelle-Guinée, a traité dans ce Supplément presque tous les sujets d'histoire naturelle » (Grand dictionnaire universel du XIXe siècle par Pierre Larousse, t. 17, deuxième supplément, Paris, Administration du grand dictionnaire universel, 1890, p. 2023).

18. Voir la nécrologie de Maurice Maindron dans le Larousse mensuel illustré, op. cit.

19. Haeckel, Arabische Korallen, op. cit., p. 2-9, 9 et 32.

20. Ibid., p. 29, 30, 43, 45.

21. Ibid., texte principal, p. 32-35 ; note $n^{\circ} 48$, p. 43 ; „Erklärung der Farbendruck-Tafeln“, „Tafel

III. Arabische Korallenbank bei Tur am Sinai“, p. 45-46.

22. Ibid., p. 43. Traduction française : «L'éclat merveilleux et incomparable des récifs de corail arabes - qu'aucun peintre ne saurait rendre - se présente très différemment selon le temps. On le voit dans toute sa somptuosité vers midi, par un temps complètement clair et calme, quand le soleil de midi brillant passe librement à travers l'eau claire comme du cristal et quand les animaux-coraux, très gonflés par l'eau, ont déployé leurs corps mous au maximum dans une complète tranquillité. Si, par contre, la lumière entre avec un angle plus aigu, si l'eau est trouble ou quelque peu en mouvement et si, par conséquent, les animaux-coraux se sont contractés et ont émis de l'eau, l'effet de lumière enchanteur est loin d'être aussi beau. »

23. Voir Hufnagel, Wissen und Diskurshoheit, op. cit., p. 208-2012.

24. Haeckel, Arabische Korallen, op. cit., p. 32-33. „Denn hier unten in der blauen Tiefe ist eigentlich Alles mit bunten Blumen überhäuft und alle diese zierlichen Blumen sind lebendige Korallenthiere. Ja sogar das bunte Moos, das die Zwischenräume zwischen den grossen Stöcken ausfüllt, zeigt sich bei genauerer Betrachtung aus Millionen winziger Korallenthierchen gebildet." Cette phrase semble avoir inspiré directement la « mousse » du vers 6 dans le sonnet.

25. Ibid., p. 33.

26. Ibid., p. 33. Traduction française : «Parmi les poissons, c'est avant tout l'étrange poule d'eau [...] qui nous frappe. Son corps plat, à forme de faucille, qui, selon l'angle d'incidence, tantôt brille d'un lustre jaune-verdâtre de bronze, tantôt d'un somptueux bleu, se termine en une longue nageoire dorsale triangulaire en haut et une analogue nageoire anale en bas. »

27. Par exemple, l'illustration réunit presque tous les éléments du vers 6 , sous la forme d'un corail de la classe des madrépores, et pour la plupart dans les mêmes couleurs évoquées dans le poème: en rouge, pendant que le madrépore est d'un jaune brunâtre et reste donc comparativement pâle. Dans le texte de Haeckel, on rencontre les mots «Moos" (mousse), «Anemone» (anémone), «Seeigel» (oursin), «purpurrot» (pourpre), « Madrepore» (madrépore), autour desquels le deuxième quatrain est construit. 
28. La dimension esthétique personnelle peut même primer la dimension scientifique : ainsi, Haeckel écrit à propos de son illustration: „Diese Tafel soll ein ungefähres Bild von der Farbenpracht und dem bunten Thierleben einer arabischen Korallenbank, nahe bei Tur, geben. [...] Ich habe mich bei dieser Skizze vorzugsweise bemüht, den unvergesslichen Eindruck und namentlich den wunderbaren Lichteffect einigermassenwiederzugeben, welchen ich selbst beim Unterrauchen und bei der submarinen Wanderunge durch eine solche Korallenschlucht in der Nähe von Tur genossen habe. [...] Zoologische Genauigkeit beansprucht diese Skizze nicht.“ (Haeckel, Arabische Korallen, op. cit., p. 45).

29. Voir Hufnagel, Wissen und Diskurshoheit, op. cit., p. 212.

30. Leconte de Lisle, Euvres complètes, t. 4, Poèmes tragiques - Les Érinnyes - Derniers poèmes L'Apollonide, éd. Edgard Pich, Paris, Champion, 2014, p. 592.

31. Revue des deux mondes, 15 octobre 1888, p. 941-942. Leconte de Lisle est reçu à l'Académie en 1887. Yann Mortelette note qu'il publie régulièrement ses poèmes dans la Revue des deux mondes à partir de cette année 1888 (voir Yann Mortelette, Histoire du Parnasse, Paris, Fayard, 2005, p. 359-360).

32. Leconte de Lisle, CEuvres, t. 3, Poèmes tragiques - Derniers poèmes, éd. Edgard Pich, Paris, Les Belles Lettres, 1977, p. 277-278.

33. Pour la définition classique du locus amoenus et de ses éléments, voir Ernst Robert Curtius, Europäische Literatur und lateinisches Mittelalter, Tübingen/Basel, Francke, ${ }^{11} 1993$, p. 202-206.

34. Rémy de Gourmont, "M. de Heredia et les poètes parnassiens ", in id., Promenades littéraires, t. 2, Paris, Mercure de France, 1963, p. 129-130.

35. Alphonse de Lamartine, "Le Lac», in CEuvres poétiques, éd. Marius-François Guyard, Paris, Gallimard, 1963, p. 38-40, ici p. 38.

36. Lamartine, « Le Lac », op. cit., p. 38, 39, 40 (v. 3, 33-34, 61-64).

37. Voir Marc Föcking, „Contre la pôhësie. Destruktion und Rekonstruktion des Poetischen in Flauberts ungeschriebener Lyrik“, in Klaus W. Hempfer (dir.), Sprachen der Lyrik. Von der Antike bis zur digitalen Poesie, Stuttgart, Steiner 2008, p. 399-428, ici p. 401-402.

38. Leconte de Lisle, Poèmes barbares, éd. Claudine Gothot-Mersch, Paris, Gallimard 1985, p. 187.

39. Ibid., p. 179.

40. Ibid., p. 149-150.

41. Lamartine, « Le Lac», p. 39-40.

42. «Je m'occupe maintenant de géologie et de botanique. C'est une chose intéressante. [...] Nous mêlerions ainsi un peu de science à nos pièces de poésie ", écrive-t-il déjà en 1839 dans une lettre (Bernard Guinaudeau, Leconte de Lisle. Premières poésies et lettres intimes, Paris, Fasquelle, 1902, p. 100, cité d'après Caroline de Mulder, Leconte de Lisle, entre utopie et république, Amsterdam, Rodopi, 2005, p. 333).

43. Jean-Louis Cabanès, " Les valeurs du vivant au tournant des $\mathrm{xIX}^{\mathrm{e}}$ et $\mathrm{xx}^{\mathrm{e}}$ siècles ", Romantisme, $\mathrm{n}$ - 154, 2011-4, p. 105-122.

44. Émile Zola, Le Docteur Pascal, in : id., CEuvres complètes, t. 6, éd. Henri Mitterand, Paris, Cercle du livre précieux, 1967, p. 1242.

45. Voir, supra, ma première contribution à ce dossier. Voir à propos des arbres de Haeckel Theodore W. Pietsch, “The trees of Ernst Haeckel, 1866-1905”, Trees of life: a visual history of evolution, Baltimore, J. Hopkins University Press, 2012, p. 98-122. Jean-Louis Cabanès lui-même fait un lien entre l'arbre du Docteur Pascal et l'arbre de Haeckel de la Generelle Morphologie. Cet arbre de Haeckel va des monères jusqu'aux races humaines (Jean-Louis Cabanès, « Les valeurs du vivant au tournant des $\mathrm{XIX}^{\mathrm{e}}$ et $\mathrm{XX}^{\mathrm{e}}$ siècles ", op. cit., p. 113).

46. Wilhelm Bölsche, Die naturwissenschaftlichen Grundlagen der Poesie. Prolegomena einer realistischen Ästhetik (1887), éd. Johannes J. Braakenburg, München, DTV, 1976, p. 34-37: „Tief unten an den Wurzeln dieses riesigen Lebensbaumes zeigt er [der Naturforscher] ihm [dem Dichter] die einfache Zelle. “ En parlant explicitement de „Fortschritt“, «progrès » et de 
„colossale Entwickelungen“ (p.36), Bölsche arrive au but: „Der Mensch ist der höchste und vollendetste Zellenstaat“ (p. 37).

47. Par exemple, les crocodiles « qui claquent des dents » ou l'hippopotame qui « ronfle, et de ses pattes grasses / Mêle la vase infecte à l'écume». Seuls les panthères et les lions font exception : ils « viennent boire » au lac.

48. Voir Henning Hufnagel, Wissen und Diskurshoheit, op. cit., p. 173.

49. Il serait intéressant d'analyser dans ce contexte de manière plus approfondie les rapports (de continuité) qu'entretient Haeckel avec la philosophie de la nature du romantisme allemand (sur Haeckel et cette philosophie les indices chez Bernhard Kleeberg voir „Evolutionäre Ästhetik. Naturanschauung und Naturerkenntnis im Monismus Ernst Haeckels“, in Renate Lachman/ Stefan Rieger (dir.), Text und Wissen. Technologische und anthropologische Aspekte, Tübingen, Narr, 2003, p. 153-179, ici p. 155-156). Alexander Nebrig constate que, à différence du naturalisme français, le naturalisme littéraire en Allemagne continue aussi à se référer à cette tradition philosophique allemande et souligne que Haeckel est une figure qui, par son œuvre, renforce cette référence (Alexander Nebrig, „Entbindung von der Disziplin. Arno Holz’ Begründung des Lebenswissens im Phantasus“, in Henning Hufnagel/Olav Krämer (éd.), Das Wissen der Poesie. Lyrik, Versepik und die Wissenschaften im 19. Jahrhundert ; Berlin/Boston, de Gruyter, 2015, p. 239-262, ici p. 246-247).

\section{ABSTRACTS}

I examine two poems, "Le Récif de Corail" by José-Maria de Heredia and "Le Lac" by Leconte de Lisle and show how they reuse and re-functionalise motifs of biological knowledge - motifs that play an important role also in Haeckel's works: The poets give them completely different meanings, realising a poetics of artistic virtuosity or engaging in a poetological debate against Romanticism. I show that, in this way, Leconte de Lisle's "Le Lac" - a poem until now completely neglected - is a meticulous repeal of Lamartine's "Le Lac".

Cet article montre comment "Le Récif de Corail» de José-Maria de Heredia et «Le Lac » de Leconte de Lisle reprennent et re-fonctionnalisent des motifs de savoir biologique, importants aussi dans l'œuvre de Haeckel : les poètes leur donnent des valeurs complètement différentes pour réaliser une poétologie de la virtuosité, hostile à toute dimension didactique, qui vise à émerveiller les lecteurs avec ses artifices, ou pour mener un débat poétologique contre le romantisme. De cette manière, l'article montre que «Le Lac » de Leconte de Lisle - poème passé jusqu'à maintenant complètement inaperçu par la critique - présente un retournement méticuleux du « Lac » de Lamartine.

\section{INDEX}

Keywords: Lisle (Leconte de), Heredia (José-Maria de), biological knowledge, poetics, Haeckel (Ernst)

Mots-clés: Lisle (Leconte de), Heredia (José-Maria de), savoirs biologiques, poétologie, Haeckel (Ernst) 
AUTHOR

HENNING HUFNAGEL

Université de Freiburg im Breisgau/Université de Zürich 